\title{
Juventud y trauma psicosocial en El Salvador
}

\author{
Nelson Portillo'
}

\section{Introducción}

El Salvador se encuentra, en la actualidad, a ocho años de la finalización de un conflicto bélico que heredó miles de muertos, lisiados y desaparecidos, y que produjo un extenso movimiento migratorio hacia dentro y fuera del país. Sin embargo, han sido ocho años que por sí solos no han borrado las secuelas psicosociales y aun algunas de las causas estructurales de dicho conflicto, las cuales todavía estarían acompañando a este pequeño país centroamericano de diversas formas.
Los gobiemos de posguerra, por su parte, se han empeñado en afirmar y resaltar hasta la saciedad su esfuerzo e incansable trabajo a favor de la consecusión de los acuerdos de paz, firmados en México, en 1992, cuando lo hecho hasta la fecha constituye únicamente la base necesaria, pero no suficiente, para una verdadera transformación social, política y económica de las condiciones histórico-estructurales prevalentes antes, durante y después de la década de los ochenta; es decir, las mismas que dieron lugar al conflicto armado (Krämer, 1998; Paige, 1998).

1. Psicólogo y analista del Instituto Universitario de Opinión Pública (IUDOP). El autor agradece a Miguel Cruz, Antonieta Beltrán y María Santacruz por sus valiosas sugerencias sobre versiones anteriores del presente trabajo. 
El número de víctimas de la guerra civil es un dato desconocido, pero ciertamente supera las cifras inexactas y conservadoras hasta ahora vertidas, debido a que los efectos fueron sentidos, directa o indirectamente, por la mayor parte de la población salvadoreña. Muchas de las víctimas sufrieron tanto lesiones físicas como psicológicas, pérdidas materiales como personales, cuando no la muerte como producto de la violencia bélica y/o represiva. Otros tuvieron que abandonar el país o se vieron forzados a desplazarse dentro de él para poder salvar su única pertenencia: su vida (Aron, 1990; Mahler, 1995; Montes, 1986). En ese contexto, a la mayoría de los salvadoreños les tocó vivir una realidad impuesta desde la cruęldad y la deshumanización de la guerra, convirtiéndose ésta en su más totalizante y cotidiano referente social (Cruz, 1997; Martín-Baró, 1990a, 1996b).

Irremediablemente, las nacientes generaciones de jóvenes, niños y niñas compartieron la fatídica experiencia de sus padres, pero con la desventaja de poseer menos recursos con los cuales afrontar tan aplastante realidad. Dicha vivencia adquiere dimensiones más pemiciosas cuando se habla de los niños como agentes activos de esa realidad, empuñando las armas o dando apoyo logístico en el campo de batalla (Cruz, 1997; IUDOP, 1999). Lo anterior corresponde al fenómeno del niño solda$d o$, el cual no fue ajeno a la guerra civil salvadoreña, según lo señalan diferentes estudios (Barnitz, 1999; Brett y McCallin, 1998; Fundación 16 de Enero, 1995; Goodwin-Gill y Cohn, 1994; Henríquez, 1994; IUDOP, 1999; Leskinen, 1996; Lindsay-Poland, 1989; Rädda Barnen, 1996; UNICEF, 1996; Woods, 1990). Particularmente uno de ellos (Fundación 16 de Enero, 1995) informa que, al final del conflicto armado, aproximadamente $-y$ sólo visto desde la perspectiva de uno de los bandos en contienda - un 20 por ciento de los ex combatientes guerrilleros tenían menos de dieciocho años; es decir, eran menores de edad.

Como era de esperar, el período de posguerra planteaba grandes expectativas y retos a todo nivel. En el caso particular de la psicología en El Salvador, la ausencia de estudio e intervención, con muy pocas excepciones, sobre los efectos psicosociales de la guerra, hizo que ésta fuera de poca ayuda en la recuperación social y psicológica de la población afectada por dicho conflicto, delatándose así como un campo semi-inoperante y poco comprometido con las grandes mayorías salvadoreñas.

Ignacio Martín-Baró, en su momento, trabajó extensamente sobre los efectos psicosociales de la guerra civil salvadoreña y llegó a dilucidar un nuevo enfoque ${ }^{2}$ como respuesta y explicación teósica al conflicto, esfuerzo que se vio acompañado por la formulación de nuevos conceptos, como lo es el de trauma psicosocial (Martín-Baró, 1990a, $1990 b$; Becker, Morales y Aguilar, 1994; ComasDíaz, 1998). No obstante, su tarea se vio truncada al convertirse él mismo en un mártir más de los tantos que el terrorismo de Estado cobró durante la guerra.

Partiendo de sus presupuestos teóricos y tomando como punto central su concepto de trauma psicosocial, se hace un análisis de la situación de guerra experimentada por jóvenes ex combatientes del Frente Farabundo Martí para la Liberación Nacional (FMLN) y de la Fuerza Armada de El Salvador. Dicho análisis se basa en la estudio Niños soldados. Lecciones aprendidas, realizado por el Instituto Universitario de Opinión Pública (IUDOP) de la Universidad Centroamericana "José Simeón Cañas" (UCA), entre los meses de diciembre de 1998 y enero de 1999, bajo el auspicio del Fondo de las Naciones Unidas para la Infancia (UNICEF). Los objetivos principales del estudio consistían en recopilar información sobre los procesos de participación bélica, la desmovilización e integración a la vida civil de ex combatientes menores de edad al término del conflicto armado, así como también explorar el impacto psicosocial que esta vivencia tuvo en ellos. En general, los resultados obtenidos sugieren que diversos efectos asociados al pasado conflicto armado se manifiestan actualmente en los planos psicológico-emocional, físico y social de los jóvenes ex combatientes.

La discusión sobre dos conceptos fundamentales ocupa el primèr apartado de este artículo: el de "niño soldado" y el de "trauma psicosocial". El primer concepto es delineado con el objetivo de

2. Como producto de su fecunda producción teórica, Martíngaró formuló lo que ahora se concibe como la "psicología [social] de la liberación", la cual continúa atrayendo a diversos autores en la actualidad. Ver, por cjemplo, las compilaciones de Aron y Corne (1996), Blanco (1998) y Pacheco y Jiménez (1990), y los trabajos de ComasDiaz (1998) y Montero (1998). 
proporcionar una idea clara sobre los protagonistas del presente trabajo ${ }^{3}$, mientras que el segundo, el de trauma psicosocial, es más extenso en contenido y se contrasta con otros conceptos paralelos - como el de estrés postraumático- con el fin de definir los alcances explicativos del concepto acuñado por Martín-Baró. De manera complementaria, en la siguiente sección se contextualiza el concepto de trauma psicosocial en el caso de los niños afectados por conflictos armados. A continuación, el tercer apartado incluye una breve reseña metodológica de la investigación en cuestión. El cuarto punto se ocupa de las experiencias de guerra que les acontecieron a los jóvenes entrevistados, mientras que el quinto se centra en la discusión de dichas vivencias desde el acervo teórico de Martín-Baró y del trauma psicosocial. Finalmente, se presentan algunas reflexiones y conclusiones sobre el tema.

\section{Aproximaciones conceptuales}

\subsection{Aproximación al concepto de "niño soldado"}

De acuerdo con Martín-Baró (1990a), el nin̄o salvadoreño se enfrentó durante la guerra a tres dilemas existenciales dentro de las tareas necesarias para su desarrollo, entendidos éstos en términos de: acción-huida, identidad-alienación y polarización-desgarramiento. Para el caso, es importante retomar el primero -el de acción-huidapor su relación con el concepto de "nin̄o soldado". Según Martín-Baró, este primer dilema determinaba dos formas no excluyentes de participación infantil en el conflicto, pudiendo ser éstas: la participación activa en él y/o la victimización a manos del mismo.

Las motivaciones de los niños que vivieron dicho dilema desde su primera forma fueron variadas y surgieron como una respuesta frontal e ineludible ante la situación de guerra que los tenía acorralados. En otros casos, la militancia de muchos otros niños y niñas estuvo más allá de sus. convicciones al haber sido reclutados por uno u otro grupo armado. Sin embargo, todos ellos compartieron, al final, un común denominador de vida, independientemente del bando y de las razones que los condujeron a participar en el conflicto: ser niños soldados. Por niño soldado se entenderá

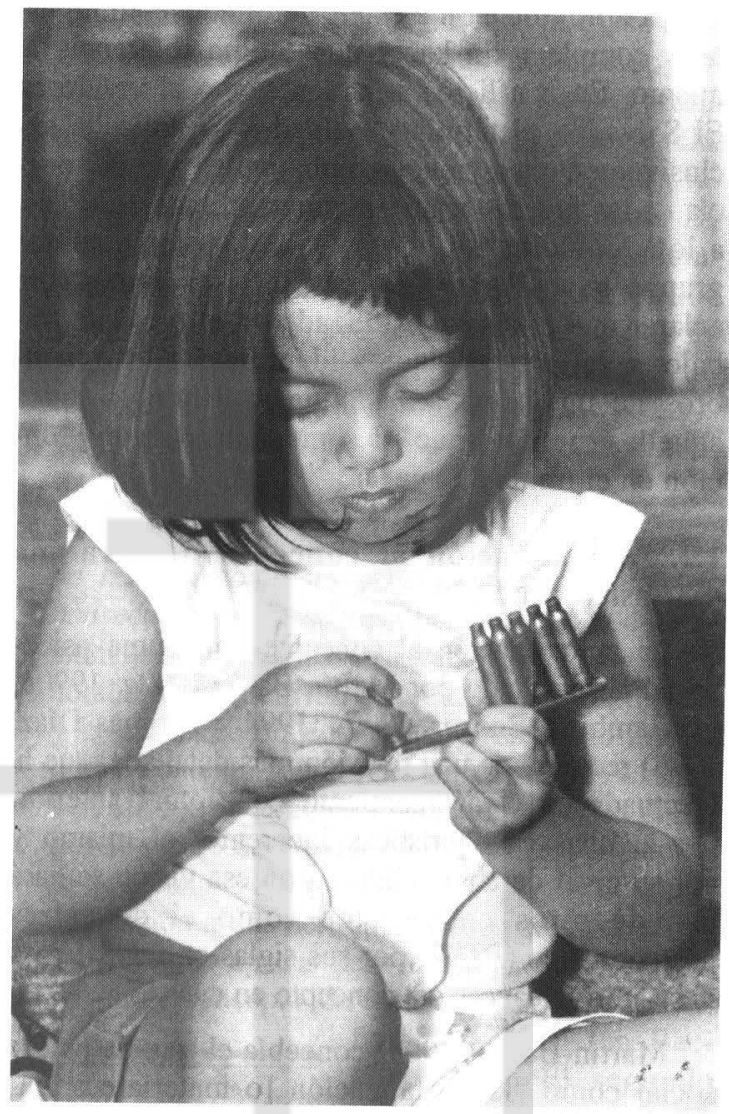

toda persona del sexo femenino o masculino, menor de 18 años de edad, que forma o ha formado parte activa, de manera voluntaria o involuntaria, de alguna organización gubernamental o de cualquier otra agrupación no gubernamental, eñ un conflicto armado, desempeñando cualquier actividad dentro de la misma. Dichas actividađes no se limitarían a las acontecidas en combate, sino que incluirían otras funciones, como la de cocineros, porteras y/o mensajeros o cualquier otra incorporación con propósitos sexuales. Además, el término también abarcaría a todos aquellos niños y niñas que se encuentran en un determinado grupo armado, a raíz de la militancia de los miembros de su familia (Bamitz, 1999; IUDOP, 1999; Rädda Barnen, 1998-1999; UNICEF, 1997). En general, se hablará de niños soldados cuando se haga alusión a menores de edad que han participado en distintas actividades en el interior de un conflicto bélico.

3. Aunque en la actualidad la mayor parte de los jóvenes entrevistados dejaron de ser niños, niñas o adolescentes, se utilizará el término para referirse al período evolutivo en el que se dio su vivencia de guerra. 
La aproximación a este primer concepto no estaría completa sin hacer una última referencia al mismo. En conflictos armados, como el vivido en El Salvador, el término "soldado" se aplicaba exclusivamente a aquellos combatientes que pertenecían a las fuerzas armadas gubernamentales, no así a los que militaban en cualquiera de los distintos grupos guerrilleros. Por tal razón es importante aclarar que el término utilizado en el presente trabajo se refiere, más que a identificar a los miembros de un grupo armado determinado, a todos aquellos menores que desempeñaban alguna función en el frente de guerra.

\subsection{Aproximación al concepto de "trauma psicosocial"}

La aproximación al concepto de trauma psicosocial desarrollado por Martín-Baró (1990a, 1990b; ver también Becker et al., 1994 y Comas-Díaz, 1998) requiere de una revisión más detallada, que la efectuada en el acercamiento conceptual anterior, por algunas características inherentes al mismo y por su nivel de complejidad. Con esa visión se hará uso de otros constructos como el de estrés postraumático ( $P T S D$, por sus siglas en inglés) para ilustrar los alcances del concepto en cuestión.

Martín-Baró (1990a) concebía el trauma psicosocial como "la cristalización [o materialización] en los individuos de unas relaciones sociales aberrantes y deshumanizadoras como las que prevalecen en situaciones de guerra civil" (p. 236). A la vez, enfatizaba que la principal cualidad de dicho concepto es el carácter dialéctico que lo constituye; es decir, el trauma es generado socialmente, pero su efecto es experimentado según la extracción social, el nivel de participación o la cercanía con el conflicto, la personalidad y experiencia de cada persona en particular (Martín-Baró, 1990b). En otras palabras, y en consecuencia, el trauma no tiene un efecto uniforme o mecánico en toda la población y constituye, en esencia, un producto sociohistórico. Esto ulteriormente condiciona dos aspectos adicionales: sus formas de comprensión y solución, las cuales se encuentran enquistadas en las misma estructuras sociales y no en el individuo, y su extensión temporal y traumatizante, las cuales están supeditadas al mantenimiento de determinadas relaciones sociales de guerra, mediatizadas por grupos, instituciones o individuos en el interior de la sociedad (Martín-Baró, 1990a). No obstante y a pesar de los efectos perniciosos atribuibles al trauma psicosocial, Martín-Baró (1990a) no descartaba que, en ocasiones excepcionales, las experiencias de guerra también pueden contribuir "al crecimiento y superación de las personas" (p. 236) mediante el ejercicio de actos prosociales como lo son, por ejemplo, la solidaridad y la cooperación (Martín-Baró, 1996a, pp. 299-357).

De esta manera, se intenta abandonar las visiones clínica, producto del modelo médico, y sociologista sobre el concepto de trauma, las cuales no logran integrar la dimensión psicológico-social de los efectos causados por la guerra y la represión política en un marco histórico determinado (Becker et al., 1994). Aunque algunos autores (Gaborit, 1999) han mostrado cuán flexible es el concepto de trauma psicosocial, al traslaparlo en contextos específicos con el de estrés postraumático, éste último no deja de carecer de ciertas particularidades del primero y presentar algunos inconvenientes de orden ideológico y diagnóstico, como se menciona más adelante. En otros casos (Becker, 1995; Becker et al., 1994; Comas-Díaz, 1998) se ha incorporado la visión de trauma psicosocial al estudio de las consecuencias psicosociales de la violencia y la represión política en latinoamérica, señalándose a la vez, particularmente Becker (1995), las deficiencias del estrés postraumático (PTSD) cuando se aplica a dichas experiencias ${ }^{4}$.

Con el fin de determinar con mayor precisión tales deficiencias, se citan las siguientes críticas:

(a) El estrés postraumático omite o hace referencia vaga al agente traumatizador. El estrés postraumático otorga igual peso a cualquier situación que pueda ser denominada como estresor. Para efectos de diagnóstico, poco interesa si la persona tuvo un accidente automovilístico o si fue torturada (ver Becker, Castillo, Gómez, Kovalskys y Lira, 1988; Simpson, 1995). Esa falta de diferenciación es peligrosa, según Becker y otros (1994), debido a que per-

4. Otros autores (Mcfarlane, 1995; Simpson, 1995; Summerfield, 1995; Yehuda y McFarlane, 1995) han señalado de igual forma diferentes inconvenientes ideológicos, históricos y políticos del concepto de cstrés postraumático, en contexlos de represión política o en otros contextos. Para una aproximación histórica al estrés postraumático, ver Kolb (1993). 
mite un disloque entre las dimensiones sociopolítica e individual, y porque la ausencia de "definición de la situación traumática impide entender la relación estrecha existente entre sintomatologia y contexto social" (p. 78). Por ejemplo, el hecho de que un joven se haya incorporado a la guerrilla después de que el ejército asesinara a sus padres, desarrollando posteriormente un cuadro sintomatológico, queda completamente al margen del concepto de estrés postraumático. Además, al no determinarse la fuente traumatizadora, se adopta una posición ideologizada que beneficia a las estructuras de poder, las cualès están interesadas en no ser identificadas o incriminadas en hechos de violencia política.

(b) Los efectos asociados a eventos traumáticos no se reducen a la dimensión temporal y sintomatológica sugeridas por el estrés postraumático. Aunque, por lo general, los efectos de las traumatizaciones ocurren a corto plazo, la probabilidad de que éstos se presenten en un plazo mayor también queda abierta, lo cual dificulta el nexo explicativo entre trauma y síntoma. En consecuencia, perder de vista la génesis de los síntomas puede sugerir un diagnóstico inexacto o equivocado del cuadro sintomatológico. Adicionalmente, los síntomas que una persona puede presentar no se reducen necesariamente a los mencionados por el estrés postraumático (Becker et al., 1994; McFarlane, 1995).

(c) El estrés postraumático entiende el trauma como una experiencia unipersonal o individual. El estrés postraumático es incapaz de explicar el alcance de la magnitud del trauma a nivel de estructuras grupales o sociales (Becker et al., 1994). La ausencia del componente grupal o social permite explicar parcialmente la vivencia traumática de una persona, ya que no se toman en cuenta los efectos concomitantes que permitirían traumatizaciones secundarias, $o$ la inclusión de personas ajenas a la experiencia traumática, a una dinámica creada por ésta misma. En el caso de una persona que ha sido torturada y amenazada de muerte, ésta se vería afectada directamente por dicha experiencia, pero ésta también puede afectar a su propia familia, la cual teme la muerte de la primera. De esta manera, la dinámica familiar y sus miembros se verían afectados por el clima de tensión y temor infundado por la experiencia de uno de ellos. Adicionalmente, esa vivencia podría entorpecer la relación de la familia con estructuras sociales mayores que pudieran comprometer su seguridad.

(d) El estrés postraumático entiende la experiencia de trauma como un proceso acompañado por consecuencias. Desde esa perspectiva, la dimensión acumulativa es soslayada, al igual que la condición cualitativa del proceso que lo produce y la constante dialéctica que existe entre individuo y contexto social (Becker et al., 1994; Becker, 1995). El carácter acumulativo, por su parte, puede ser de dos tipos. En el primero, el trauma es procesual al ser ocasionado por la continua exposición a una serie de sucesos que, por sí solos, no son capaces de evocarlo, lo cual corresponde al concepto de trauma acumulativo acuñado por Khan (en Becker, 1995). El segundo tipo de efecto acumulativo sostiene que si se da una secuencia de vivencias traumáticas, éstas tenderían a cronificar los efectos producidos por las mismas (Keilson, en Becker, 1995). Adicionalmente, según Del Solar y Piper (en Becker et al., 1994), la cronificación también se vería reforzada en el caso que las condiciones que las provocaron persistan a través del tiempo.

(e) El concepto de estrés postraumático no cubre conceptualmente la transmisión generacional de experiencias traumáticas. Como afirman Becker y otros (1994), el hecho de que el estrés postraumático no sea flexible en el manejo del factor tiempo y que no logre capturar procesos, implica una serie de limitantes al momento de establecer conexiones nosológicas con segundas, terceras o cuartas generaciones. 
(f) El concepto subyacente al estrés postraumático implica la imposibilidad total o parcial de prevención de la experiencia traumática. Esta visión no puede ser menos que engañosa en contextos de conflicto sociopolítico, como sucedió en El Salvador, Chile, Brasil, África del Sur y otras muchas latitudes, en donde se desarrollan actos deliberados de violencia represiva y organizada contra la población (Lira y Weinstein, 1990; Martín-Baró, 1990a; Murillo, 1990; McFarlane, 1995; Simpson, 1995; Summerfield, 1995).

De todo lo anterior se derivan algunos puntos finales en el afinamiento del concepto de trauma psicosocial. En primer lugar, está la primacía del contexto social en la construcción histórica de experiencias traumáticas, que afectan diferencialmente a sectores de la sociedad. Un segundo punto estabablece que la "herida" psicológica producida es un factor importante dentro de los efectos del trauma psicosocial, pero su injerencia no acaba en dicha dimensión ni en la individualidad de la persona misma. Finalmente, y en tercer lugar, el carácter dialéctico inherente al trauma psicosocial exige un análisis y un ajuste estructural, en el caso que se deseen realizar evaluaciones o intervenciones sobre sus efectos.

\section{Trauma psicosocial y nin̄ez}

El sentido amplio y comprehensivo propio del concepto del trauma psicosocial hay que rastrearlo en la noción de salud mental que Martín-Baró (1990c) sostenía. Según su planteamiento, la salud mental no constituye fundamentalmente un estado psicológico interno cuanto un reflejo de las relaciones sociales mediatizadas en un contexto histórico específico ${ }^{5}$. En el caso particular de la guerra salvadoreña, señalaba que ésta había socavado seriamente las bases de la convivencia humana y que los efectos se revelarían diferencialmente en distintos sectores de la sociedad, como fue sugerido en el concepto de trauma psicosocial. De ese modo, las manifestaciones asociadas a la vivencia del trauma, ya sea como síntomas individuales o distorsiones en las relaciones sociales, constituirían una respuesta "normal" a la "anormalidad" del carácter deshumanizador de las relaciones humanas que la guerra habría institucionalizado.

Cabe resaltar que de los grupos afectados por el conflicto armado, a Martín-Baró le preocupaba especialmente el de los niños por su vulnerabilidad. Como se mencionó, los niños salvadoreños se vieron enfrentados a dilemas existenciales (ver MartínBaro, 1990a), los cuales restringían y suponían serios obstáculos para su sano desarrollo psicosocial. Los "hijos de la guerra" fraguaron su identidad y su personalidad en un contexto donde la violencia, la desconfianza, la polarización, la persecución y el miedo eran elementos propios de la convivencia diaria (Becker et al., 1994). En el caso de los niños soldados, otras experiencias se sumaban a las muchas que una buena parte de los niños salvadoreños se encontraba viviendo.

Martín-Baró (1990c) señalaba que muchos de los efectos de la guena en la niñez salvadoreña aparecerían de manera inmediata a la vivencia del conflicto. Sin embargo, enfatizaba que muchos otros concomitantes iban a darse también a mediano y largo plazo. Para el caso, diversos síntomas o manifestaciones psicológicas constituirían respuestas "lógicas" asociadas a eventos traumatizantes. Gaborit (1999), por ejemplo, sostiene que los elementos más comunes relacionados con traumatizaciones extremas son: (1) síntomas psicológicos (ansiedad, depresión y disociación), (2) trastornos psicosomáticos, (3) alteraciones en la excitación fisiológica, (4) deficiencias en procesos cognitivos y (5) dificultades en el establecimiento de relaciones interpersonales. Estos elementos son importantes, ya que permiten establecer el impacto del evento traumatizante de manera individual, pero aislados son tan poco informativos y útiles sobre la experiencia traumática como lo es, en sí mismo, el concepto de estrés postraumáticos.

El hecho de que la salud mental y, más específicamente, el trauma psicosocial sean productos

5. Sisti (1995) reconoce la aguda intuición de Martín-Baró, al proponer éste la calidad de las relaciones sociales como principio fundamentador de la salud mental. De hecho, Sisti coloca dicho elemento como el eje central de su propuesta de salud mental de base.

6. Como señala Gaborit (1999), no fuc hasta después de mediados de los ochenta que los síntomas asociados al estrés postraumático en niños fucron incluidos en el Manual diagnóstico y estadístico de los trastornos mentales, de la Asociación Psiquiátrica Americana (DSM-III-R) (Ver Diagnostic and Statistical Disorder Manual of Mental Disorders. Washington, DC: American Psychiatric Association, 1987). Para una revisión de las respuestas postraumáticas en los niños, ver también Schwarz y Perry (1994). 
dialécticos entre la persona y la sociedad en la que ésta se desenvuelve, supone que los efectos van más allá de su enraizamiento individual y se acuerpan en la disrupción de las relaciones sociales. En tal sentido, muchos niños no sólo sufrirían los embates psicológicos y emocionales de la guerra, sino que también enfrentarían el desaparecimiento de sus comunidades, la desintegración de sus grupos familiares, la postergación de etapas de su desarrollo, la pérdida de figuras de apego e identidad, e innumerables procesos de duelo sin resolver (ver Becker et al., 1994; Gaborit, 1998, 1999). Adicionalmente, Gaborit (1998) sostiene que experiencias tan extremas como éstas inciden de modo negativo en la preciudadanía, es decir, en "las representaciones mentales de los niños, sus actitudes $y$ valoraciones afectivas que tienen relación con el sistema político -y su sustentación" (p. 497).

En resumen, los efectos asociados al conflicto bélico suponen serios condicionantes para el desarrollo psicosocial de los niños y graves secuelas durante su adultez, además de limitantes a futuro en la calidad de las relaciones humanas al interior del tejido social y las posibilidades de desarrollo de un sistema social con vocación democrática (ver, Djeddah, 1996; Gaborit, 1998, 1999).

\section{Metodología}

\subsection{Muestra}

La investigación Niños soldados. Lecciones aprendidas, realizada por el IUDOP y financiada por UNICEF, contó con la participación de 293 jóvenes, cuyas edades estaban comprendidas entre los 17 y 25 años de edad. La mayor parte de ellos declararon haber nacido entre 1976 y 1979 —58 por ciento de la muestra-, lo que significa que al momento de la firma de los acuerdos de paz, tenían una edad promedio de 15 años. Del total de la muestra, el 70 por ciento pertenece al sexo masculino, mientras que el 30 por ciento restante pertenece al sexo femenino. Según el grupo armado al cual pertenecían, los datos recopilados muestran que el 95 por ciento, es decir 278 jóvenes, perteneció a las filas del FMLN, mientras que el resto, 15 jóvenes, se encontraba enlistado en el ejército salvadoreño. En el caso de las jóvenes encuestadas, cabe resaltar que todas pertenecieron exclusivamente al FMLN. Otros datos demográficos declarados muestran que los jóvenes ex combatientes contaban con una edad promedio de 12 años de edad cuando iniciaron su participación en el conflicto armado.

La encuesta se llevó a cabo en 61 comunidades o cantones distribuidos en 31 municipios, pertenecientes a 11 departamentos de la república salvadoreña (Cuadro 1). Debido a la distribución de la muestra se puede afirmar que los datos recopilados tienen una mayor representatividad estadística para jóvenes que combatieron en cualquiera de los grupos que se aglutinaron bajo el FMLN, que para los jóvenes que combatieron en la Fuerza Armada. No obstante, la información aportada sólo por el 5 por ciento de la muestra, el cual representa la vivencia de menores de edad de la FAES, tiene una riqueza cualitativa que, más allá de su validez estadística, sirve de contrapunto en el análisis de los resultados del estudio.

\section{Cuadro 1}

Número de municipios y comunidades visitadas, según departamento y total de casos recolectados

\begin{tabular}{lcrrr}
\hline Departamento & Muni- & Comu- & \multicolumn{2}{c}{ Casos } \\
& cipios & nidades & \multicolumn{1}{c}{ N } & \multicolumn{1}{c}{$\%$} \\
\hline Chalatenango & 8 & 17 & 97 & 33.1 \\
Usulután & 2 & 10 & 43 & 14.7 \\
San Vicente & 2 & 7 & 42 & 14.3 \\
Cuscatlán & 3 & 11 & 34 & 11.6 \\
Cabañas & 2 & 2 & 29 & 9.9 \\
Morazán & 4 & 8 & 29 & 9.9 \\
San Salvador & 3 & 9 & 11 & 3.8 \\
La Paz & 3 & 3 & 3 & 1.0 \\
Sonsonate & 2 & 2 & 3 & 1.0 \\
La Libertad & 1 & 1 & 1 & 0.3 \\
San Miguel & 1 & 1 & 1 & 0.3 \\
\hline Totales & 31 & 61 & 293 & 100.0 \\
\hline
\end{tabular}

Fuente: elaboración propia, con base en los datos del IUDOP, 1999. 


\subsection{Instrumento}

El instrumento utilizado para estudiar el fenómeno de los niños soldados fue diseñado conjuntamente por el equipo técnico del IUDOP y representantes de UNICEF. Dicho instrumento contaba con secciones dirigidas exclusivamente a ex combatientes de la Fuerza Armada o del FMLN, y otras partes de éste eran comunes a ambos grupos. La versión final, avalada por UNICEF, estaba constituida por 132 reactivos, los cuales cubrieron nueve temáticas. El estudio ejecutado es por demás extenso y rico en información (ver IUDOP, 1999). No obstante, el presente trabajo se centra principalmente en aquellas áreas que recopilaban datos geográficos y sociodemográficos relacionados con el entrevistado, información sobre su participación en el conflicto armado, discapacidades sufridas a raíz del mismo, procesos e indicadores de reintegración social e impacto psicosocial causado por la guerra.

\subsection{Procedimiento}

La definición de la muestra de jóvenes ex combatientes que pertenecieron al FMLN y a la Fuerza Armada, en el presente estudio, se estableció siguiendo un proceso multietápico. En primer lugar, mediante un muestreo intencional se estipuló que quienes pertenecieran a ambos grupos debían ser seleccionados de acuerdo con dos criterios fundamentales: haber participado en el conflicto armado realizando cualquier actividad que los vinculase con alguno de los grupos armados mencionados, y haber sido menor de edad cuando se firmaron los acuerdos de paz o, en su defecto, contar con una edad no mayor de 24 años en el momento de la realización del trabajo de campo. Paralelamente se había establecido una cuota determinada para el grupo del FMLN, según el sexo de los entrevis- tados de dicho grupo sobre la base de un estudio previo (SRN, en Leskinen, 1996). Luego se identificaron las comunidades por visitar a través de la información previamente recopilada, contactos personales y comunicación directa con entidades relacionadas con el tipo de población que se perseguía encuestar. Esto permitió establecer, geográficamente, los lugares donde se concentraban asentamientos considerables de personas que tomaron parte en el conflicto armado, o que fueron refugiados durante el período en que éste tuvo lugar?.

Para la aplicación del cuestionario se contó con la orientación de miembros de las comunidades, o se procedió a visitar aleatoriamente cada unidad habitacional, con el fin de localizar de manera directa a los jóvenes. Adicionalmente se visitaron algunos lugares, como canchas de fútbol, celebraciones religiosas o sembradillos. Una vez identificados los jóvenes que cumplian con los requerimientos, se les explicaban los objetivos y la temática del estudio para luego realizar la entrevista, si el joven seleccionado deseaba colaborar. En el caso de aquellos que pertenecieron a la Fuerza Armada, se procedió de distinta manera. En este se contó con la colaboración de organizaciones relacionadas con ex combatientes del ejército. Mediante dos de ellas se entrevistó a un pequeño grupo de ex soldados discapacitados y en proceso de rehabilitación, que llenaban los requisitos estipulados en el estudio.

\section{Participación y vivencia del conflicto armado}

Un recorrido elemental, pero tentativamente completo, de la participación de los jóvenes ex combatientes en el conflicto armado debería explicar cómo y por qué se incorporaron, qué actividades les fueron encomendadas y cuáles fueron los

7. Esto fue aplicado fundamentalmente para seleccionar a los jóvenes que pertenecieron al FMLN, debido a que las pocas comunidades pobladas por ex efectivos de la Fuerza Armada estaban compuestas esencialmente por aquellos que salieron beneficiados con el Programa de Transferencia de Tierras y que, por ende, habían sido mayores de edad cuando los acuerdos de paz fueron firmados. Esto último significó operativamente la sobreponderación de uno de los grupos respecto al otro. 
efectos físicos, psicológicos y sociales producidos por su militancia. A continuación se desarrollan en detalle dichos planteamientos.

\subsection{La incorporación}

Barnitz (1999), con base en los estudios realizados por Brett y McCallin (1998), señala que la existencia de diversos factores o condiciones tienden a incrementar la probabilidad de que los niños se incorporen o sean incorporados en un conflicto armado. Ella menciona específicamente cinco condiciones: (1) vivir en extrema probreza, (2) la pérdida de los padres o del grupo familiar, (3) la ausencia de lazos estrechos dentro de la familia, (4) residir en zonas de combate, y (5) la destrucción de la ecología inmediata del niño (escuelas, casas, hospitales, etc.). En el caso de la experiencia salvadoreña, el estudio sobre niños soldados, realizado por el IUDOP, permite determinar que dichos condicionantes fueron experimentados en diferentes grados por los jóvenes entrevistados.

En primer lugar, el estudio señala que los jóvenes ex combatientes, en su mayoría, vivían y continúan viviendo en condiciones de extrema pobreza, como la mayor parte de la población que habita en el interior del país. Los resultados revelan que alrededor de la mitad de los jóvenes entrevistados no cuentan con ingresos propios, lo cual en gran parte puede deberse a su corta edad. Sin embargo, el 82 por ciento de los que mencionaron tener algún tipo de ingreso, dijeron percibir menos de un salario mínimo mensual. La situación de pobreza no viene dada sólo por los mínimos ingresos que perciben, sino también por el número de personas que dependen de ellos. Un 47.8 por ciento de los jóvenes mencionaron que vivían con una pareja, mientras que el 58.7 por ciento mencionó que tenía hijos. Más aún, de los que tienen hijos, un 76.7 por ciento ha procreado uno o dos, mientras que el resto tiene tres o más. A esto se le agrega el elevado número de personas que conviven en la misma vivienda, el cual asciende a un promedio de cinco a seis, según lo declarado por los jóvenes entrevistados.

En segundo lugar, muchos de los jóvenes entrevistados dijeron haber pérdido a sus padres o demás familiares durante la guerra, lo cual implicó

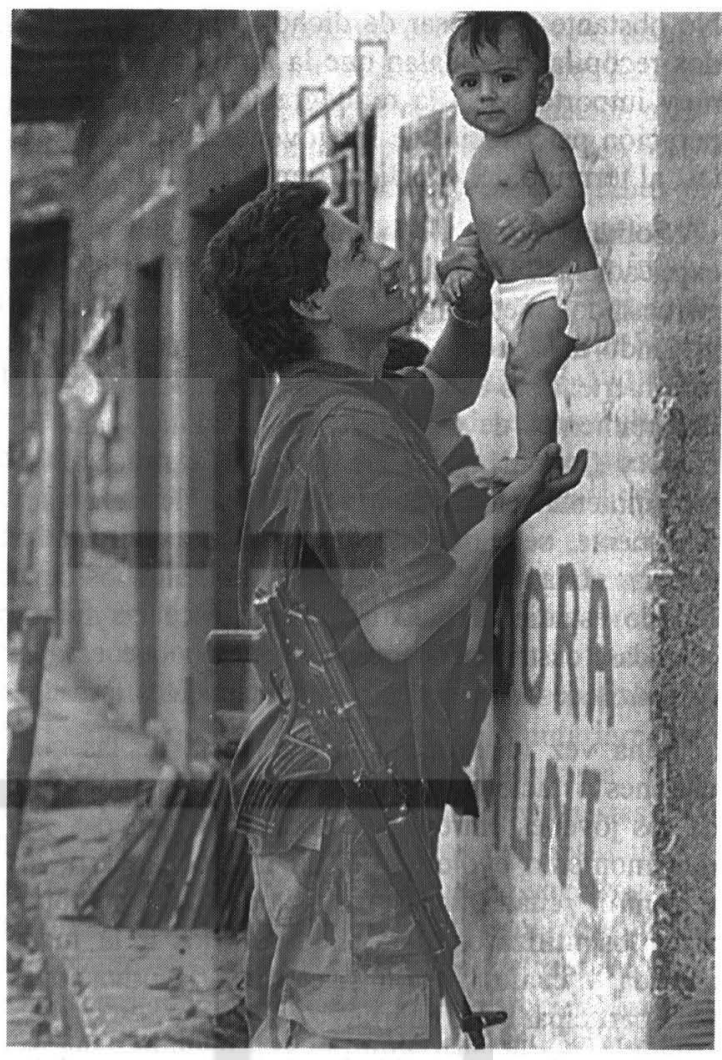

su posterior militancia. Aunque muchos lo expresaron de manera abierta, otros mencionaron lo mismo durante las entrevistas independientemente de la razón asociada a su incorporación. Es decir, aunque la muerte de los padres u otros parientes no haya sido la razón citada, la ocurrencia de la misma incrementaba las posibilidades para que luego se convirtieran en niños soldados.

Sobre el tercer condicionante que Barnitz plantea - grado de cohesión familiar durante el conflicto-, no es posible determinar completamente su injerencia debido a la falta de medición de dicho constructo. Aunque el estudio sobre niños soldados señala que más de la mitad de los jóvenes entrevistados - el 54 por ciento- experimentaron un cambio en la estructura de su grupo familiar después de la guerra, es difícil establecer con precisión cuál era el grado de cohesión que mantenían con su grupo familiar previo y, aún más, las verdaderas causas que provocaron dicho cambio ${ }^{8}$.

8. La guerra, como factor desintegrador de la familia, es ampliamente válido como se ha mencionado anteriormente; sin embargo, muchos de los cambios pueden deberse esencialmente a cambios esperables en la vida del adulto (tener pareja, hijos, etc.) - lo cual se refleja en muchas de las respuestas dadas por los jóvenes participantes-o a una combinación de ambos elementos. 
No obstante y a pesar de dicho vacío, los resultados recopilados señalan que la familia tuvo un rol muy importante en la reintegración social y recuperación psicosocial de los jóvenes ex combatientes, al término del conflicto armado".

Sobre el cuarto y el quinto factor existen algunas explicaciones. Una parte considerable de los jóvenes encuestados mencionaron haber vivido o continúan viviendo en aquellas zonas donde la guerra golpeó más fuerte. Esto es, en poblados pertenecientes a los departamentos de Chalatenango, Usulután, San Vicente y Cuscatlán, por ejemplo. La destrucción de los ambientes en los que los niños se desenvolvían, finalmente, se dio como resultado de vivir en las mismas zonas de combates. Más aún, algunos dejaron de estudiar debido a que sus escuelas fueron cerradas, destruidas o porque tuvieron que escapar (guindas) de bombardeos o enfrentamientos armados.

Una vez identificada la existencia de las condiciones o factores de riesgo vivenciados por parte de los jóvenes entrevistados, cabe preguntarse, ien qué momento evolutivo de su niñez la guerra los reclamó?, ¿cuáles fueron los métodos que los grupos armados utilizaron para incorporarlos a la lucha armada y cuáles fueron sus propias motivaciones para participar en la guerra?

El estudio Niños soldados. Lecciones aprendidas encontró que la mayor parte de los jóvenes entrevistados que participaron en el conflicto armado se incorporaron aun siendo bastante jóvenes. Como se mencionó en su momento, la edad promedio de incorporación es 12 años aproximadamente, es decir, en los arbores de su adolescencia. No obstante, se estableció que el $\mathbf{4 8}$ por ciento informó haberse incorporado a la lucha armada cuando tenía menos de 12 años. De hecho, se dieron casos en los cuales las incorporaciones sucedieron desde los 7 años de edad. La mayoría de tales incorporaciones se dieron durante los últimos años del conflicto, lo cual resulta lógico tomando en cuenta la corta edad de dicha generación de jóvenes. Específicamente, el año que registra mayor número de incorporaciones es 1988, mientras que algunas otras se dieron en 1989 , en el marco de la ofensiva general lanzada por el FMLN a finales de dicho año.

Si los datos sobre la edad de incorporación resultan sorprendentes, las razones que los motivaron
- u obligaron- a participar en la guerra no lo son menos. Como en la mayor parte de conflictos armados, los combatientes de cada uno de los grupos armados eran obtenidos de dos formas: mediante incorporaciones voluntarias o a través de operativos de reclutamiento. En general, el estudio encontró una tendencia mayor de incorporación voluntaria entre los jóvenes que pertencieron al FMLN, que entre aquellos que formaron parte de la Fuerza Armada. De los que dijeron haber pertencido al FMLN, el 91.7 por ciento declaró haberse enlistado de forma voluntaria, mientras que el 8.3 por ciento restante dijo haber sido reclutado. En el caso de los jóvenes que formaron parte del ejército salvadoreño, más de la mitad —el 53.3 por ciento- dijo que fue reclutado, aunque una parte considerable -l 46.7 por ciento- declaró haberse incorporado voluntariamente.

Respecto a los niños salvadoreños que fueron reclutados tanto por el FMLN como por la Fuerza Armada, resulta interesante hacer algunos apuntes a la luz de otros estudios. En primer lugar, los datos sobre reclutamiento contradicen lo que algunos autores (Goodwin-Gill y Cohn, 1994) habían expresado sobre la práctica de reclutamiento de menores por parte del FMLN, la cual se suponía que había terminado en 1986, según autoridades del mismo grupo armado. En segundo lugar, aunque los resultados referentes a los jóvenes de la Fuerza Armada no gozan del beneplácito de la representatividad estadística, muestran que el porcentaje de menores reclutados era alto. De hecho, otras fuentes (Leskinen, 1996; UNICEF, 1996) han señalado que año con año las trincheras del ejército eran atiborradas con una considerable cuota de menores de edad. Un dato que tiende a apoyar la tesis de reclutamientos masivos de menores de edad era el promedio de nuevos soldados requeridos anualmente. Cada año la FAES reclutaba entre 12 y 20 mil jóvenes, entre campesinos y pertenecientes a estratos pobres (Brett y McCallin, 1998), quienes tenían que cumplir un periodo de servicio militar obligatorio de 2 años. No obstante, de los soldados reclutados, sólo un 25 por ciento continuaba después que dicho período había terminado, lo que perpetuaba los operativos de reclutamiento (Leskinen, 1996). Un tercer y último punto está relacionado con la coerción y la violencia con la cual los reclutamientos eran realizados. En el

9. Becker et al. (1994) encontraron también que el tema de la familia como elemento de recuperación era un punto recurrente e importante en un estudio realizado con niños, niñas y adolescentes sobre los efectos de violencia sociopolítica, en distintos países latinoamericanos. 
caso del FMLN, aunque éste tendía las más de las veces a echar mano de procesos de inducción, en algunas ocasiones se llevaba a los jóvenes que encontraba a su paso por los poblados, ya sea mediante amenazas o reclamos a las familias residentes (Goodwin-Gill y Cohn, 1994). En el caso del ejército, el reclutamiento forzoso solía ir acompañado de altas dosis de violencia y coerción por parte de los efectivos que realizaban dichos operativos (Santiago, 1993, pp. 27-36).

Retomando la discusión de las tendencias de incorporación, es importante identificar cuáles fueron las motivaciones que dieron pie a los enlistamientos voluntarios. Acorde con los resultados obtenidos, las principales razones que mediatizaron dichas incorporaciones pueden ser divididas en tres grupos básicos: en razones ideológicas, razones personales y razones familiares (Cuadro 2). En el primer grupo, los jóvenes esgrimían haberse incorporado para que las condiciones de vida de la población mejorasen, para defender a la patria, para instaurar una sociedad más justa o porque querían ayudar a la causa mediante su participacipación. Un segundo conglomerado de razones -las personales - plantea que algunos jóvenes participaron en la guerra porque les gustaba o porque era su fuente de trabajo. Finalmente, un tercer grupo de razones estaba ligado a realidades un tanto más concretas, como son las familiares. La participación de muchos jóvenes, en tal sentido, se debió a la pérdida del grupo familiar - lo que concuerda con lo mencionado por Bamitz-, a la militancia de miembros de su familia, al aliento de otras personas cercanas o al deseo de vengar la muerte de parientes asesinados.

\section{Cuadro 2}

Tipo de razón por la cual el joven se incorporó a la guerra

\begin{tabular}{lrr}
\hline & $\mathrm{N}^{*}$ & $\%$ \\
\hline Razones ideológicas & 164 & 62.6 \\
Razones personales & 52 & 19.8 \\
Razones familiares & 46 & 17.6 \\
\hline Total & 262 & 100.0 \\
\hline
\end{tabular}

* Sólo incluye a aquéllos que se incorporaron voluntariamente.

Fuente: elaboración propia en base a datos del IUDOP, 1999.

Ahora bien, aun cuando cada una de estas motivaciones puede significar el fiel reflejo de los sen- timientos y las convicciones de cada joven, resulta obvio que en el interior de algunas de ellas se desdibuja un fuerte componente de indoctrinación o inducción, el cual terminaría por empujar a los jóvenes a incorporarse. Cabe comentar que tanto el FMLN como la Fuerza Armada se encargaron de endosar su proyecto de guerra a la población infantil, tomando ventaja de los factores de riesgo que ésta experimentaba. Otros estudios (Martín-Baró, 1989) confirmarían dicha tesis. Martín-Baró (1989), en un estudio realizado con niños de estratos altos y bajos, en zonas poco afectadas por la guerra, encontró que un 31.4 por ciento de los niños consultados deseaban ser soldados o combatientes, lo cual constituía - según él- un indicador del grado de militarización de la mente de los niños salvadoreños. Dicha disposición infantil a incorporarse a la lucha armada contrasta sensiblemente con el alto porcentaje de jóvenes que se incorporaron voluntariamente (89.4 por ciento) registrado acá. No obstante, Martín-Baró enfatizaba que el patrón de respuestas podía ser distinto en aquellos niños que residian en zonas de guerra. Este hallazgo no sólo sugeriría que los niños que habitaban zonas de guerra estaban efectivamente más expuestos a los factores de riesgo, lo cual condicionó su posterior participación bélica, sino también que la militarización de las mentes y los corazones de tales niños fue evidentemente mucho mayor.

Los resultados revelan así mismo que las razones de enlistamiento militar toman un matiz diferente en función del grupo al cual pertenecieron los jóvenes ex combatientes. En el caso de los jóvenes que pertenecieron al FMLN, las razones más frecuentemente citadas fueron las ideológicas, mientras que en el caso de los jóvenes que lucharon del lado de la Fuerza Armada las más comunes fueron las razones personales, como el gusto por combatir o porque era su fuente de trabajo.

Las distinciones hechas anteriormente entre los grupos de razones tendrían implicaciones más allá de su función explicativa, según lo sugieren diversos estudios. Algunos autores (Henríquez, 1994; Punamäki, 1996) consideran que la apreciación o compromiso ideológico con la guerra es una variable moderadora del impacto que ésta tendría posteriormente en la persona. En el caso de los niños soldados, éstos habrían estado, hasta cierto punto, mejor preparados para afrontar la guerra que los demás niños, debido a la elaboración ideológica que podían construir sobre ella. No obstante, Henríquez (1994) 
señala que los efectos derivados de la guerra serían determinados ulteriormente por dos variables adicionales -el grado de contacto con el conflicto y el contexto social en el que se experimentaba dicha vivencia- y, más concretamente, por las posibles permutaciones entre las tres.

En una línea similar, Marans, Berkman y Cohen (1996) plantean que la ideología puede funcionar como un factor protector y que el impacto causado por la exposición a la violencia está mediatizado por el universo de significados que éstos atribuyan a los eventos externos (Garbarino, Kostelny, Dubrow, 1991a). Otros autores (Goodwin-Hill y Cohn, 1994; Macksoud, Aber y Cohn, 1996) señalan que la elaboración ideológica de la guerra u otros procesos de significación son asequibles, en la medida en que se haya alcanzado cierto grado de desarrollo cognitivo como para manejar conceptos abstractos.

De manera interesante, el estudio realizado por el IUDOP tiende a corroborar la correlación entre la edad y la elaboración ideológica de la guerra. Según el Cuadro 3, las motivaciones ideológicas fueron realizadas por los jóvenes que contaban con mayor edad cuando se incorporaron a la lucha armada, en comparación con aquellos que tenían menos años de edad. Sin embargo, las razones personales también tenderían a ser elaboradas por aquellos que contaban con mayor edad. En el caso de los nin̄os que se incorporaron por razones familiares, es probable que, por su corta edad, éstos dependieran más de su entorno, lo cual no les permitía tomar sus propias decisiones, además de que no contaban con un desarrollo cognitivo suficiente para elaborar significaciones más abstractas de su misma incorporación como aquellos niños que poseían un mayor número de años.

\section{Cuadro 3}

Edad de enlistamiento, según el tipo de razón por el cual el joven se incorporó a la guerra

\begin{tabular}{ll}
\hline & Edad* $^{*}$ \\
\hline Razones ideológicas & 12.32 \\
Razones personales & 11.92 \\
Razones familiares & 10.78 \\
\hline
\end{tabular}

* Promedios.

Fuente: elaboración propia con basc en los datos del IUDOP, 1999.

\subsection{Las actividades}

El estudio sobre los niños soldados salvadoreños sugiere que no sólo las razones de su militancia fueron diversas, sino también las funciones o actividades que les fueron delegadas durante la guerra. Los datos de la investigación revelan que la principal función que tuvieron que desempeñar los jóvenes siendo niños fue la de combatir. Así lo confirmó uno de cada dos entrevistados (50.9 por ciento) al referirse a la actividad a la cual se dedicaron durante el conflicto armado ${ }^{\prime \prime}$. No obstante, otro buen número de jóvenes mencionó que la actividad a la cual se dedicaba consistía en conducir mensajes o información, lo cual se conocía como "correo". Esta segunda función fue mencionada por el 19.8 por ciento de los jóvenes encuestados; es decir, uno de cada cinco jóvenes. Del resto de funciones mencionadas, una tercera estaba más bien relacionada con actividades de tipo logístico, lo cual implicaba transportar armamento o apoyar cualquier otra iniciativa en el frente de guerra.

Las actividades mencionadas hasta ahora mantuvieron ocupados durante la guerra relativamente al 80 por ciento de los jóvenes encuestados, en tanto que el resto llevó a cabo otra tríada básica de funciones. Los datos compilados señalan que un 7.5 por ciento de jóvenes tenían bajo su responsabilidad elaborar los alimentos, un 5.8 por ciento tenía asignada la tarea de manejar las operaciones de comunicaciones, mediante equipos de radio, y un 4 por ciento se desempeñó como sanitario, es decir, hacía las veces de enfermero cuidando la salud de los de su grupo.

Un segundo acercamiento a las actividades señaladas sugiere que muchas de ellas estaban asociadas directamente con el sexo y la edad de cada niño. Por ejemplo, la funciones de combatiente, "correo" y apoyo logístico eran asignadas sobre todo a los jóvenes del sexo masculino. Sin embargo, tomando las edades de incorporación mencionadas como puntos de referencia, se puede inferir que las misiones de combate eran dadas sobre todo a los que contaban con más años, mientras que la tareas de "correo" y asistencia logística eran del dominio de los más chicos. Por otro lado, cocinar, manejar equipos de comunicación (radista) y ocuparse de la salud de los demás eran funciones más propias de las niñas y las adolescentes. Además, estas niñas tendían a incorpo-

10. La categoría de combatiente se aplica tanto para los jóvenes que pertenecieron al FMLN, como a los que formaron parte de la Fuerza Armada. El resto de las categorías se aplican únicamente a los que combatieron en el FMLN. 
rarse, teniendo una mayor edad, sobre todo las que se encargaban de cocinar.

Los datos sobre la distribución de las funcio- nes se presentan a continuación, en el Cuadro 4, en porcentajes generales, por sexo y edad de incorporación al grupo armado en el cual combatieron los jóvenes entrevistados.

\section{Cuadro 4}

Funciones principales desempeñadas por los niños soldados en porcentajes generales, por sexo y por edad de incorporación

\begin{tabular}{lrrrrr}
\hline Función & $\mathrm{N}$ & $\%$ & \multicolumn{2}{c}{ Porcentajes por sexo } & Edad de \\
\cline { 4 - 5 } & & & Masculino & Femenino & incorporación* \\
\hline Combatiente & 149 & 50.9 & 82.8 & 17.2 & 9.74 \\
Correo & 58 & 19.8 & 84.6 & 15.4 & 13.03 \\
Logístico & 34 & 11.6 & 55.9 & 44.1 & 10.65 \\
Cocinera & 22 & 7.5 & 17.6 & 82.4 & 12.53 \\
Comunicaciones (radista) & 17 & 5.8 & 0.0 & 100.0 & 16.05 \\
Sanitario & 13 & 4.4 & 0.0 & 100.0 & 11.85 \\
\hline
\end{tabular}

* Promedios.

Fuente: elaboración propia con base en los datos del IUDOP, 1999.

\subsection{Los efectos}

Probablemente el mayor peso del balance negativo de la militancia de niños en conflictos armados recae sobre los hombros de los efectos que esto conlleva. El estudio Niños soldados. Lecciones aprendidas, realizado por el IUDOP, indagó la magnitud de los efectos que la guerra produjo en los niños soldados salvadoreños a nivel psicológico, físico y social.

A nivel psicológico, los resultados asociados a la guerra se develan por sí solos. Después de ocho años de haber finalizado el conflicto armado, la mayor parte de los jóvenes reporta respuestas emocionales y psicológicas relacionadas con su experiencia militar. Se habla de relación debido a la inviabilidad para determinar grados de causalidad entre respuestas y experiencias de guerra, tanto por la brecha que separa la investigación con la finalización del conflicto como también por la existencia de eventuales vulnerabilidades personales; sin embargo, se asume que muchas de ellas están fuertemente correlacionadas con el haber vivido la guerra como agentes activos dentro de la misma y con el momento evolutivo en el que esta experiencia tomó lugar.

En primer lugar, los resultados presentados en el Cuadro 5 revelan una cierta propensión a enfermarse: el 19.1 de los jóvenes entrevistados afirmó enfermarse "siempre" o "casi siempre", mientras que cuatro de cada diez (el 40.6 por ciento) di- jeron enfermarse "algunas veces". Claro está que el nexo entre esta propensión y su experiencia militar está modulada por factores que van desde la

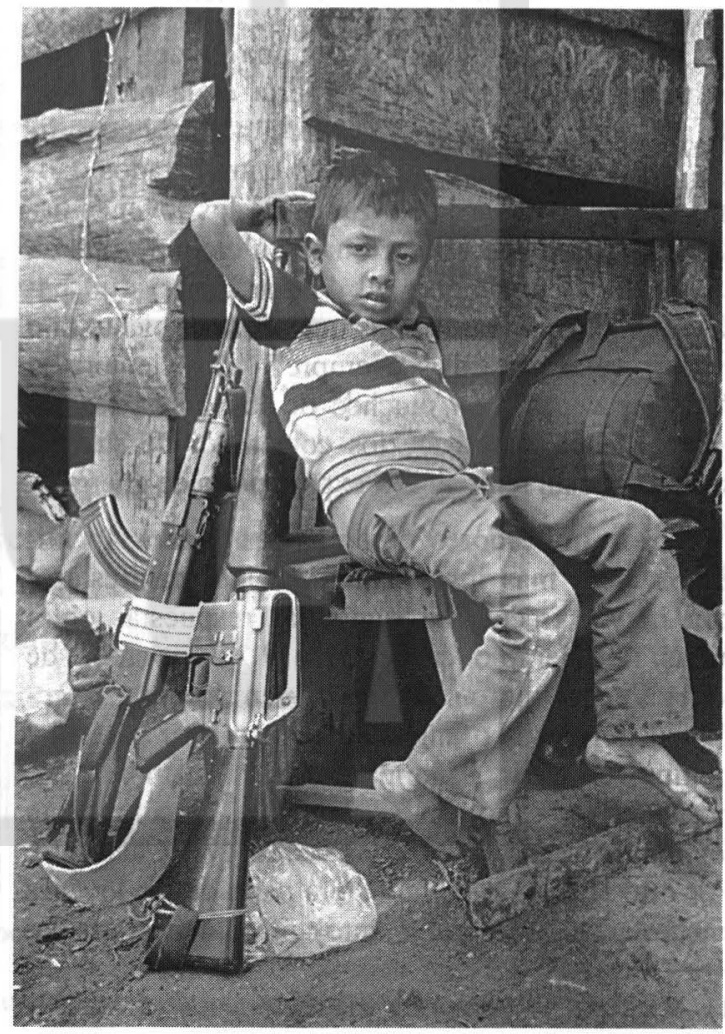


mala alimentación hasta la falta de servicios básicos y médicos, todos males asociados a la pobreza, como también por afecciones enraizadas en el mismo conflicto (lesiones o heridas de guerra, por ejemplo). Los altos porcentajes de propensión a enfermarse obtenidos resultan interesantes, si se toma en cuenta que la mayoría de los jóvenes no pasa de los 24 años de edad - sobre el presupues- to de que a dicha edad no existe deterioro masivo en los principales sistemas orgánicos-. No obstante, algunos autores (Davison y Pennebaker, 1996) han establecido que existe cierta relación entre experiencias traumáticas y propensión a desarrollar enfermedades y problemas de salud en general, lo cual podría sugerir un nexo entre su estado de salud actual y sus experiencias de guerra.

\section{Cuadro 5}

Respuestas emocionales y psicológicas presentadas por los jóvenes entrevistados*

\begin{tabular}{lcccc}
\hline Indicadores & Siempre & Casi siempre & Algunas veces & Total \\
\hline Padece de enfermedad & 6.5 & 12.6 & 40.6 & 59.7 \\
Padece de insomnio & 8.9 & 11.3 & 20.8 & 41.0 \\
Padece de pesadillas & 6.1 & 9.9 & 29.7 & 45.7 \\
Se siente nervioso/a & 14.8 & 22.0 & 34.0 & 70.8 \\
Se siente angustiado/a & 7.2 & 19.8 & 39.2 & 66.2 \\
Se enoja con facilidad & 21.5 & 15.0 & 26.6 & 63.1 \\
Se siente cansado/a y deprimido/a & 21.2 & 17.4 & 35.8 & 74.4 \\
Piensa en lo sucedido & 39.6 & 18.8 & 25.6 & 84.0 \\
\hline
\end{tabular}

" No incluye las opciones de respuesta "nunca" y "casi nunca".

Fuente: tomado y adaptado por el IUDOP, 1999.

Lo que sí parece constituir una evidencia más palpable del impacto de la guerra es la presencia de una serie de indicadores emocionales y cognitivos registrados por los jóvenes entrevistados. Como revela el Cuadro 5, algunos de los jóvenes padecen algún grado alteración en su ciclo normal de sueño. Específicamente, el 41 por ciento dijo padecer de insomnio, mientras que 45.7 por ciento dijo experimentar pesadillas "siempre", "casi siempre" o "algunas veces". En muchos de los casos, los jóvenes mencionaban que los contenidos de sus pesadillas hacían referencia a experiencias de guerra".

Otros indicadores emocionales menos focalizados, pero más importantes, suelen manifestarse con mayor frecuencia según las respuestas obtenidas. Por ejemplo, casi el 37 y el 27 por ciento de los jóvenes dijeron sentirse -en forma respectiva - nerviosos o angustiados la mayor parte del tiempo, es decir, "siempre" o "casi siempre". No obstante, otros porcentajes elevados experimentan los mismos estados "algunas veces" (ver Cuadro 5). Por otro lado, más de la tercera parte de los jóvenes encuestados (36.5 por ciento) presentan cierta la- bilidad emocional, lo cual se refleja en la facilidad con la que dicen experimentar enojo.

Lo que llama fuertemente la atención es la frecuencia con la que los jóvenes abordados experimentan cansancio y diferentes grados de depresión. A pesar de lo demandante que son las labores agrestes y domésticas, a las cuales la mayoría de los consultados se dedican, es difícil creer que su estado cuasi-perenne de cansancio se deba particularmente a tales razones. Adicionalmente, dicho cansancio es acompañado por una disminución en el tono emocional, lo cual se experimentaría en las formas de desmotivación o desgano generalizado y no en grados patológicos de depresión clínica (IUDOP, 1999).

El último de los indicadores explorado señala que la mayor parte de los jóvenes se encuentran anclados en su pasado bélico: el 58.4 por ciento de los encuestados declararon que piensan "siempre" - "casi siempre" en lo que les sucedió durante la guerra, mientras que un 25.6 por ciento dijo pensar "algunas veces" en lo mismo.

La descripción separada de cada uno de los indicadores señalados resulta ser bastante ilustrativa,

11. Ver IUDOP, 1999, para una revisión más detallada. 
pero no permite capturar las interrelaciones entre ellos y otras variables, y entre los mismos. Esto se puede realizar mediante dos formas alternativas, pero complementarias. La primera viene dada por la conformación de los ocho ítemes diferentes en una sola escala, la cual mediría el impacto de la guerra mediante una serie de indicadores psicoemocionales asociados a la misma. De esa manera, las respuestas se distribuyen en una escala transformada de 10 puntos con el objetivo de proveer un medio más comprensible de dichos indicadores ${ }^{12}$. Este ejercicio estadístico señala que la escala obtenida goza de un nivel de consistencia intema relativamente alto (Alfa de Cronbach $=0.76$ ). La segunda estrategia incluye establecer el grado de asociación entre los indicadores, mediante una serie de correlaciones de Pearson.

La escala construida con los ocho ítemes, la cual puede ser llamada escala de impacto psicoemocional, señala que no existen diferencias significativas entre los jóvenes del sexo femenino y masculino, entre los que pertenecieron al FMLN y a la Fuerza Armada, entre los que fueron reclutados y los que se incorporaron voluntariamente, entre los que se incorporaron por razones ideológicas o por cualquier otra razón y entre las diferentes funciones desempeñadas durante la guerra, en lo que a indicadores psicoemocionales se refiere. Sin embargo, sí existen diferencias significativas entre aquellos que sufrieron lesiones físicas, en comparación con los que no las experimentaron $(X 2=41.95$, $p=0.03$ ), entre las diferentes edades de incorporación $(\mathrm{F}=1.94, \mathrm{p}=$ 0.02 ) y entre aquellos que experimentaron más cercanía a la violencia bélica $(\mathrm{X} 2=108.23, \mathrm{p}=0.004)$.

Respecto a la estrategia asociativa, el Cuadro 6 proporciona una matriz de correlaciones de Pearson, la cual describe distintos grados de asociatividad entre dos distintas variables de los diferentes indicadores medidos. A partir de dicha matriz vale la pena llamar la atención sobre algunas relaciones en particular. En primer lugar, la relación que existe entre alteraciones nerviosas (D) y estados de angustia (E) constituye el coeficiente más alto obtenido $(r=0.554)$. Esto indica que las personas que dicen sentirse "nerviosas" tienden también a experimentar cierto grado de angustia. Sin embargo, "sentirse nervioso" está correlacionado con sentirse cansado y deprimido (F) $(r=0.465)$, con pensar en eventos suscitados en la guerra $(G)(r=0.347)$ y con irritabilidad $(H)$ ( $r=0.386$ ). Además, experimentar angustia (E) está relacionado con estados de depresión y cansancio físico $(F)(r=0.508)$. La centralidad de las alteraciones emocionales es más fácil de apreciar cuando se observa la correlación entre sentirse nervioso (D) y angustiado (E) con el padecimiento de pesadillas $(C)(r=0.340$ y 0.405 , respectivamente). Finalmente, vale la pena mencionar que experimentar pesadillas (C) está correlacionado con problemas de insomnio $(B)(r=0.373)$.

En general, el Cuadro 6 demuestra que todos los coeficientes se encuentran correlacionados y que las correlaciones son estadísticamente significativas. Adicionalmente, el hecho de establecer el grado de asociación que un indicador tiene con otro demuestra que éstos actúan como un solo cuerpo de manifestaciones. En suma, y como hipótesis, se puede plantear que los efectos psicoemocionales de la militancia se expresan en una disrupción afectiva generalizada (angustia y nerviosismo) asociada con alteraciones del sueño (pesadillas e insomnio), estados depresivos, cansancio físico, orientación temporal hacia el pasado (pensamientos recurrentes y reminiscencias de guerra) e irritabilidad (facilidad para enojarse). Otros estudios señalan resultados similares (Arroyo y Eth, 1996; Barnitz, 1999; Henríquez, 1994; Henríquez y Méndez, 1992; Holman y Cohen Silver, 1998; Ma-

12. Originalmente, las alternativas de respuesta en los ocho reactivos estaban distribuidas en una escala de cinco punlos. Los valores asignados iban desde $(0)$, lo cual señalaba que la persona nunca experimentaba la sintomatología sugerida, hasta (4), lo cual indicaba que la persona la expcrimentaba sicmprc. 


\section{Cuadro 6}

\section{Matriz de correlaciones de los ítemes de la escala de impacto psicoemocional}

Indicadores
A
B
C
D
E
F
G

\begin{tabular}{|c|c|c|c|c|c|c|c|}
\hline B & $0.332\left({ }^{* *}\right)$ & & & & & & \\
\hline $\bar{C}$ & $0.192\left(^{* *}\right)$ & $0.373(* *)$ & & & & & \\
\hline D & $\left.0.210^{* *}\right)$ & $0.192\left(^{* *}\right)$ & $0.340(* *)$ & & & & \\
\hline E & $0.241\left(^{* *}\right)$ & $0.222\left(^{* *}\right)$ & $0.405\left(^{* *}\right)$ & $0.554\left(^{* *}\right)$ & & & \\
\hline $\mathbf{F}$ & $0.273\left(^{* *}\right)$ & $0.240\left(^{* *}\right)$ & $0.272(* *)$ & $0.465\left(^{* *}\right)$ & $0.508(* *)$ & & \\
\hline G & $0.179\left(^{* *}\right)$ & $0.119\left(^{*}\right)$ & $0.242\left(^{* *}\right)$ & $0.347\left(^{* *}\right)$ & $0.273\left(^{* *}\right)$ & $0.319\left(^{* *}\right)$ & \\
\hline H & $0.208\left(^{* *}\right)$ & $0.166\left(^{* *}\right)$ & $0.162\left(^{* *}\right)$ & $0.386\left(^{* *}\right)$ & $0.194\left(^{* *}\right)$ & $0.291\left(^{* *}\right)$ & $0.231(* *)$ \\
\hline
\end{tabular}

* $p=0.05$

${ }^{* *} p=0.01$

(A) Frecuencia con la que se enferma. (B) Frecuencia con la que padece insomnio. (C) Frecuencia con la que experimenta pesadillas. (D) Frecuencia con la que presenta alteraciones nerviosas. (E) Frecuencia con la que experimenta angustia. (F) Frecuencia con la que se siente cansado y deprimido. (G) Frecuencia con la que piensa en eventos suscitados durante la guerra. (H) Frecuencia con la que se enoja con facilidad.

rans, Berkman y Cohen, 1996; Punamäki, 1989, entre otros). Sin embargo, estos "síntomas" representan sólo algunos de los resultados psicológicos asociados a la guerra. Habría que ver cómo ese mismo episodio impactó en el desarrollo de la identidad (ver, por ejemplo, Barnitz, 1999; Becker et al., 1994; Comas-Díaz, 1998) y la moral (ver Garbarino, Kostelny, Dubrow, $1991 b$ ) en cada uno de ellos, sólo por citar dos factores importantes.

Ahora bien, los resultados revelan también que una buena parte de los jóvenes encuestados, además de haber sido afectados psicológicamente, también fueron afectados más directamente en forma física por la guerra. El estudio realizado por el IUDOP señala que un 27.6 por ciento de los jóvenes padecen de algún tipo de incapacidad auditiva, visual o física. De hecho, al preguntárseles sobre lo peor que les sucedió durante el conflicto armado, un $\mathbf{1 5 . 7}$ por ciento mencionó haber sufrido heridas o quedar incapacitado. Los resultados muestran también que sólo a un 14.8 por ciento de aquellos jóvenes que sufrieron discapacidades se les facilitó alguna prótesis o algún tipo de ayuda, mientras que el 83.7 por ciento no recibió ni terapia física ni psicológica después de la guerra.
Finalmente, es importante afirmar que los efectos de la guerra se extendieron mucho más allá de las mentes y de los cuerpos de los jóvenes entrevistados, expresándose éstos en profundas pérdidas sociales ${ }^{13}$. Por ejemplo, los niños soldados perdieron la oportunidad para formarse académicamente por haber prestado servicio militar. Antes de incorporarse a la lucha armada, los jóvenes contaban con escasos tres años y medio de formación educativa como promedio y sólo una quinta parte de ellos dijeron haber retomado los estudios después del conflicto armado. Paralelamente, los jóvenes fueron desprovistos de sus redes de apoyo social mediante la destrucción sistemática de sus comunidades y el desmembramiento de sus familias (ver Gaborit, 1998; Stroebe y Stroebe, 1996). En tercer lugar, los jóvenes ex combatientes han sufrido una profunda pauperización, lo cual ha implicado un deterioro generalizado en su calidad de vida. Como se mencionó anteriormente, la mayor parte de los jóvenes que una vez fueron niños soldados viven en condiciones de extrema pobreza. Sus ingresos son mínimos y apenas gozan de servicios básicos. Según lo manifestaron, sólo la mitad de los encuestados (50.9 por ciento) tienen acceso a servicios de salud y agua potable, mientras

13. Para una revisión más detallada de los efectos causados por la guerra en la niñez salvadoreña, revisar el excelente trabajo realizado por Gaborit (1998). 
que el resto tiene acceso sólo a uno de los dos servicios (31.7 por ciento) o a ninguno de ellos (17.4 por ciento). El estudio sugiere que sus penas también son compartidas por sus hijos. El 79 por ciento de los que mencionaron tener hijos también comentaron que ellos no tienen una adecuada alimentación, lo cual corresponde a los altos índices de desnutrición infantil informados en el interior del país ${ }^{14}$. No obstante, la asistencia en materia de salud y educación es más asequible para sus pequeños, al menos en teoría, según lo mencionado. A lo anterior hay que agregar que muchos de los jóvenes tuvieron problemas para decidir o encontrar dónde vivir, para ser aceptados por sus comunidades, para encontrar a sus familias y para recibir beneficios derivados de los acuerdos de paz, los cuales no significaron mayores ganancias para ellos (ver IUDOP, 1999).

\section{Experiencia de guerra y trauma psicosocial}

Distintos autores han mencionado o discutido reiteradamente sobre los efectos psicológicos y los procesos de socialización que los niños salvadoreños experimentaron bajo la devastadora impronta del conflicto bélico (Becker et al., 1994; Cruz, 1997; Escobar, Guandique, Pineda, Ramos, Reyes y Linares, 1991; Fernández, 1993; Gaborit, 1998; Henríquez, 1991; Henríquez y Méndez, 1992; Martín-Baró, 1989, 1990a; Rodríguez, 1987).

En el caso particular de los niños soldados, los datos proporcionados dan fe de las múltiples y profundas laceraciones que la juventud ex combatiente de El Salvador padece. En primer lugar, los resultados muestran alteraciones psicológicas y emocionales diversas. Sin embargo, desde la perspectiva del trauma psicosocial, eso corresponde únicamente a la punta del iceberg y tal vez constituye el menor de sus problemas. Fijar la mirada sólo en dichos indicadores equivaldría a ignorar sus raíces: las relaciones sociales que las provocaron. La guerra, como materialización histórica de dichas relaciones, se ensañó principalmente y en forma selectiva con uno de los grupos enfrentados, el más débil y vulnerable: el de los pobres. La historia salvadoreña demuestra reiteradamente que las grandes mayorías siempre han sido las más flageladas por los intereses de unos pocos, y la guera civil iniciada en los ochenta, lo único que hizo fue confirmarlo. Las condiciones presentadas por los niños soldados son, probablemente, un caso extremo de trauma psicosocial, producto de la misma guerra. Su extracción social, su corta edad y su cercanía con el conflicto determinó las pocas opciones de vida que tenían y los obligó a tomar parte en un conflicto construido históricamente. De hecho, es ahí cuando se dice que el trauma se configura sobre la base de un momento sociohistórico y que las experiencias derivadas afectan diferencialmente a la población. Más aún, el estudio señala que los efectos psicosociales experimentados por los niños soldados han sido tan envolventes que se dejan ver aun después de muchos años de finalizada la guerra. Lo anterior concuerda perfectamente con lo planteado por Martín-Baró $(1990 b)$, cuando éste menciona que el trauma psicosocial afecta a la persona como un todo.

Paralelamente, el presente trabajo tendería a confirmar dos tesis planteadas por el psicólogo salvadoreño. Martín-Baró (1990b) consideraba, en primer lugar, que el trauma psicosocial es una expresión normal "de un sistema social basado en relaciones sociales de explotación y opresión deshumanizadoras" (p. 236) y que éste, en segundo lugar, tiende a afectar principalmente a los niños que deben crecer en medio de dichas relaciones deshumanizadoras. Con certeza cabe decir que el fenómeno de los niños soldados logra capturar, en esencia, ambos planteamientos.

Un punto importante, que encaja de igual manera con el modelo de trauma psicosocial, es el hecho de que algunos jóvenes encuestados perciben haber crecido personalmente muy a pesar de las duras experiencias de guerra que tuvieron que vivir. Más aún, muchos jóvenes consideran que lo mejor que les sucedió durante la guerra fue haber tenido la oportunidad de luchar por el bien de las personas y las relaciones de compañerismo que los unieron con otros combatientes.

Finalmente, vale la pena llamar la atención sobre sus condiciones de vida actuales. A pesar de que la guerra ya finalizó, el saldo que ésta dejó en los jóvenes ex combatientes fue muy negativo. La guerra cercenó su infancia, su adolescencia, sus sueños, les robó a sus familias y los afectó de forma física y psicológica. No obstante, dentro de la lista

14. Ver, por ejemplo, el esludio sobre desnutrición infantil de Brentlinger, Hernán, Hernández-Díaz, Azaroff y McCall (1999), en zonas rurales, en el período de posguerra. 
de males asociados con su experiencia de guerra, la pobreza, como factor estructural, se mantiene como característica perenne y determinante de sus vidas. No sin tristeza, muchos jóvenes mencionaban que la guerra les heredó peores condiciones de vida que las que tenían antes, situación que les produce mucha frustración y resentimiento.

\section{Reflexiones y conclusiones}

Sin lugar a duda, la guerra civil salvadoreña ha sido uno de los eventos más trágicos de la historia contemporánea del país. No obstante, es justo decir que la tragedia que ésta engendró fue mucho mayor para unos que para otros. Particularmente, los sectores campesinos y los desposeídos -es decir, la mayoría de los salvadoreños - fueron los más golpeados en ese oscuro pasaje, tanto porque contribuyeron en demasía con los combatientes como con los muertos, los heridos y los desaparecidos.

Dentro de ese entramado probablemente los niños conformaron uno de los grupos más afectados por la guerra y, en especial, los que se vieron obligados a empuñar las armas en el campo de batalla $^{15}$. Las experiencias y los efectos que la guerra grabó en los niños soldados salvadoreños, es una de las expresiones más claras de las relaciones sociales "aberrantes" que posibilitaron dicho conflicto. Más aún, su experiencia encaja perfectamente con lo que Martín-Baró concibió como trauma psicosocial. Su ramplante situación de pobreza y las pérdidas que sufrieron en el camino determinaron, en buena medida, su incorporación al conflicto armado y su posterior traumatización psicológica, física y social.

Con todos estos males a cuestas, tal vez lo peor es que los jóvenes que lucharon en la guerra han quedado en el olvido. Voluntaria o involuntariamente, atraídos por anhelos de justicia o por razones de subsistencia, estos jóvenes dieron indudablemente una de las mejores partes de sus vidas sin lograr ningún cambio sustancial en ellas. Ahora, muchos tienen dificultades físicas para escuchar o mirar, mientras se los engulle la pobreza.
Sus hijos han heredado los males que la misma guerra les echó sobre sus hombros: no tienen qué comer o no asisten a la escuela. Otros tantos probablemente aplican lo aprendido en la guerra: matar. Otros, tal vez, buscan espacios donde continuar ejercitando patrones de violencia o ganar cuotas de poder, cuando no la forma de ganarse el pan de cada dia ${ }^{16}$.

Aunque el presente estudio se centró especialmente en aquellos niños que participaron en la guerra, queda pendiente estudiar lo que pasó con los que no participaron, pero que por sus mismas condiciones sociales pudieron haber sido afectados. Más aún, la pregunta puede ser extendida a los miles de adultos que también sufrieron los males de la guerra y que a la fecha podrían experimentar sus efectos. Sin embargo, esta tarea requiere del levantamiento del velo del silencio institucionalizado, impuesto desde las altas esferas del poder establecido. Dada la relación entre el trauma y el contexto sociopolítico, la elaboración de las pérdidas humanas y sociales, como también de otros concomitantes psicoemocionales, demanda el reconocimiento del daño infringido como paso inicial para la reconcialición psicosocial (Becker et al., 1994). De otra manera, las mismas relaciones "aberrantes" que se encarnaron hace poco menos de una década, continuarían actualizando o condicionando nuevos traumas psicosociales. De hecho, es allí donde radica el desafío que los psicólogos enfrentan al acompañar a los salvadoreños en el período de posguerra: estimular la "participación activa y concientizadora" de los grupos de mayor exclusión social con el objetivo de mejorar la "calidad de las relaciones sociales" $y$, consecuentemente, su salud mental ${ }^{17}$ (ver las propuestas de Sisti, 1995, y Martín-Baró, 1990d; también, Martín-Baró, $1996 b$, pp. 302-304, con respecto a la concientización de los grupos primarios).

Como punto final cabe decir que no sólo la psicología en El Salvador se encuentra frente a dicho desafío, al retomar o volver la mirada hacia una tarea que se creía superada, sino también las elites que detentan el poder en el país, ya que la

\footnotetext{
15. Algunos autores (Leskinen, 1996; Sisti, 1995) señalan que la guerra civil salvadoreña fue una guerra librada en ambos bandos por diferentes generaciones de jóvenes. Más aún, en el caso de los combatientes del FMLN, vale destacar que cran conocidos como "los muchachos", lo cual sugicre la corta edad que tenían.

16. Cruz y Portillo (1998) encontraron que el 12.2 por ciento de los jóvenes que pertencen a una pandilla, en el árca metropolitana de San Salvador, declararon haber combatido durante la gucrra.

17. Tomado y adaptado de Sisti (1995, p. 57).
} 
guerra y sus efectos están enquistados fundamentalmente en las estructuras sociales, económicas y políticas que los posibilitan.

\section{Bibliografia}

Aron, A. "Problemas psicológicos de los refugiados salvadoreños en California". En I. Martín-Baró (ed.), Psicología social de la guerra. San Salvador: UCA Editores, 1990, pp. 465-481.

Aron, A. y Shawn, C (eds.). Writings for a Liberation Psychology. Ignacio Marlin-Baró. Cambridge, MA: Harvard University Press, 1996.

Arroyo, W. y Eth, S. "Post Traumatic Stress Disorder and Other Stress Reactions". En R. J. Apfel (ed.), Minefields in Their Hearts. The Mental Health of Children in War and Communal Violence. New Haven: Yale University Press, 1996, pp. 53-74.

Barnitz, L. A. Child Soldiers: Youth Who Participate in Armed Conflict. Booklet No. 1, Washington, DC: Youth Advocate Program International, 1999.

Becker, D. "The Deficiency of the Concept of Posttraumatic Stress Disorder When Dealing with Victims of Human Right Violations". En R. J. Kleber, C.R. Figley y B. P. Gersons (eds.), Beyond Trauma. Cultural and Societal Dynamics. New York: Plenum Press, 1995, Pp. 99-110.

Becker, D.; Castillo, M. I.; Gómcz, E.; Kovalskys, J. y Lira, E. "Psicopatología y proceso psicoterapéutico de situaciones políticas traumáticas", Revista de Psicología de El Salvador, San Salvador, Vol. VII, 30, 1988, pp. 293-308,

Becker, D.; Morales, G. y Aguilar, M. I. (eds.). Trauma psicosocial y adolescentes latinoamericanos: formas de acción grupal, Santiago, Chilc: Instituto Latinoamericano de Salud Mental y Derechos Humanos (ILAS), 1994.

Blanco, A. (coord.). Ignacio Martín-Baró. Psicología de la liberación. Madrid: Trotta, 1998.

Brentlinger, P. E.; Hernan, M. A.; Hernandez-Diaz, S.; Azaroff, L. S. y McCall, M. "Childhood Malnutrilion and Postwar Reconstruction in Rural El Salvador: A Community-Based Survey", Journal of the American Medical Association, 281, 1999, pp. 184-190.

Brett, R. y McCallin, M. Children: The Invisible Soldiers. Stockholm: Rädda Barnen, 1998.

Comas-Diaz, L. "Ethnic Conflict and the Psychology of Liberation in Guatemala, Peru, and Puerto Rico", American Psychologist, 53, pp. 778-792.

Cruz, J. M. "Los factores posibilitadores y las expresiones de la violencia en los noventa", Estudios Centroamericanos (ECA), 588, 1997, pp. 977-992.

Cruz, J. M. y Portillo Peña, N. Solidaridad y violencia en las pandillas del gran San Salvador. Más allá de la vida loca. San Salvador: UCA Edilores, 1998.
Davison, K. P. y Pennebaker, J. W. "Social psychosomatics". En E. T. Higgings y A. W. Kruglanski (eds.), Social Psychology. Handbook of Basic Principles. New York: Guilford Press, 1996, pp. 102-130.

Djeddah, C. "Children and armed conflict", World Health, 49, p. 12.

Escobar, M. E.; Guandique, G. M.; Pineda, A. B.; Ramos, M.; Reyes, E. A. y Linares, C. S. "Estudio de algunos efectos psicológicos que presentan los niños que han vivido eventos de guerra", Revista de Psicología de El Salvador, San Salvador, Vol. X, 42, 1991, pp. 281-289.

Fundación 16 de Enero. Los niños y jóvenes excombatientes en su proceso de reinserción a la vida civil, San Salvador: Rädda Barnen de Suecia, 1995.

Fernández, A. "El nin̄o y el adolescente afectado por la guerra", Revista de Psicología de El Salvador, San Salvador, Vol. XII, 48, 1993, pp. 89-113,

Gaborit, M. "Psicología social de la nin̄ez en El Salvador: condiciones en la construcción de la preciudadanía", Estudios Centroamericanos (ECA), $595-$ 596, 1998, pp. 977-992.

Gaborit, M. "Aspectos psicosociales de un desastre natural: cl huracán Mitch y El Salvador", Estudios Centroamericanos (ECA), 606, 1999, pp. 351-366.

Garbarino, J.; Kostelny, K. y Dubrow, N. No Place to Be Child: Growing Up in a War Zone. Lexington, MA: Lexington Books, $1991 a$.

Garbarino, J.; Kostelny, K. y Dubrow, N. "What Children Can Tell Us About Living in Danger", American Psychology, 46, 1991b, pp. 376-383.

Goodwin-Gill, G. y Cohn, I. Child Soldiers: The Role of Children in Armed Conflicts. A Study on Behalf of the Henry Dunant Institute, Geneva. New York: Oxford University Press, 1994.

Henríquez, J. L. "Guerra y vida psicológica de niños en zonas de conflicto", Revista de Psicologia de El Salvador, San Salvador, Vol. X, 39, 1991, pp. 85 91 .

Henríquez, J. L. "Community-Based Mental Health Projects for Persons Affected by the War and Former Child Soldiers in the FMLN". En Child Soldiers: Report From a Seminar by Rädda Barnen and the Swedish Red Cross, 12-13 de febrcro, 1994, Estocolmo, Suiza (manuscrito).

Henríquez, J. L y Méndez, M. "Los efectos psicosociales de la guerra en niños de El Salvador", Revisıa de Psicología de El Salvador, San Salvador, Vol. $\mathrm{XI}, 44,1992$, pp. 89-107.

Holman, E. A. y Cohen Silver, R. "Getling "Sluck» in the Past. Temporal Orientation and Coping with Trauma", Journal of Personality and Social Psychology, 74, 1998, pp. 1146-1163.

Instituto Universitario de Opinión Pública (IUDOP). Niños soldados. Lecciones aprendidas. Informe Privado, San Salvador, 1999. 
Krämer, M. El Salvador. Unicornio de la memoria. San Salvador: Ediciones Museo de la Palabra.

Kolb, L. C. "The psychobiology of PTSD: Perspectives and reflections on the Past, Present and Future", Journal of Traumatic Stress, 6, 1993, pp. 293-304.

Leskinen, R. Child soldiers in El Salvador. Research project related to the study on Children in Armed Conflicts undertaken at the request of the United Nation. Estocolmo: Rädda Bamen, 1996 (manuscrito).

Lindsay-Poland, J. Youth Under Fire. Military Conscription in El Salvador: A Summary Report. Eureka Springs, AK: Center on War \& the Child, 1989 (manuscrito).

Lira, E. y Weinstein, E. "La tortura. Conceptualización psicológica y proceso terapéutico". En I. Martín-Baró (ed.), Psicología social de la guerra. San Salvador: UCA Editores, 1990, pp. 335-390.

Macksoud, M.; Aber, J. L. y Cohn, I. "Assesing the Impact of War on Children". En R. J. Apfel (ed.), Minefields in Their Hearts. The Mental Health of Children in War and Communal Violence. New Haven: Yale University Press, 1996, pp. 218-230.

Mahler, S. J. Salvadorans in Suburbia. Symbiosis and Conflict. Boston, MA: Allyn and Bacon, 1995.

Marans, S.; Berkman, M. y Cohen, D. "Child Development and Adaptation to Catastrophic Circunstances". En R. J. Apfel (ed.), Minefields in Their Hearts. The Mental Health of Children in War and Communal Violence. New Haven: Yale University Press, 1996, pp. 104-127.

Martín-Baró, I. "La institucionalización de la guerra", Revista de Psicologia de El Salvador, San Salvador, Vol. VIII, 33, 1989, pp. 223-245.

Martín-Baró, I. "Guerra y trauma psicosocial del niño salvadoreño". En I. Martín-Baró (ed.), Psicología social de la guerra. San Salvador: UCA Edilores, 1990a, pp. 234-249.

Martín-Baró, I. “La violencia política y la guerra como causas de trauma psicosocial en El Salvador". En I. Martín-Baró (ed.), Psicología social de la guerra. San Salvador: UCA Editores, 1990b, pp. 65-84.

Martín-Baró, I. "Guerra y salud mental". En I. MartínBaró (ed.), Psicología social de la guerra. San Salvador: UCA Editores, 1990c, pp. 23-40.

Martín-Baró, I. "La violencia en Centroamérica: Una visión psicosocial", Revista de Psicología de El Salvador, San Salvador, Vol. IX, 35, 1990d, pp. 123 146.

Martín-Baró, I. Acción e ideología. Psicologia social desde Centroamérica (8a. Edición). San Salvador: UCA Editores, $1996 a$.

Martín-Baró, 1. Sistema, grupo y poder. Psicología social desde Centroamérica (II) (3a. Edición). San Salvador: UCA Editores, $1996 b$.

McFarlane, A. C. "The Severety of the Trauma. Issues About Its Role in Posttraumatic Stress Disorder". En R. J. Kleber, C. R. Figley y B. P. Gersons (eds.),
Beyond Trauma. Cultural and Societal Dynamics. New York: Plenum Press, 1995, pp. 31-54.

Montero, M. "Retos y perspectivas de la psicología de la liberación. Consideraciones a finales de siglo", Estudios Centroamericanos (ECA), 601-602, 1998, pp. 1123-1135.

Montes, S. "El problema de los desplazados y refugiados salvadoreños", Estudios Centroamericanos (ECA), 447-448, 1986, pp. 37-58.

Murillo Perdomo, A. "Psicoterapia con víctimas de la tortura". En I. Martín-Baró (ed.), Psicología social de la guerra. San Salvador: UCA Editores, 1990, pp. 317-333.

Pacheco, G. y Jiménez, B. (eds.). Ignacio Martín-Baró (1942-1989). Psicología de la liberación para América Latina. Guadalajara: Instituto Tecnológico y de Estudios Superiores de Occidente, Departamento de Extensión Universitaria, Universidad de Guadalajara, 1990.

Paige, J.M. Coffee and Power. Revolution and Rise of Democracy in Central America. Cambridge, MA: Harvard University Press, 1998.

Punamäki, R. L. "Respuestas de stress psicológico de las madres palestinas y sus hijos a las condiciones te ocupación militar y violencia política". En I. MartínBaró (ed.), Psicología social de la guerra. San Salvador: UCA Editores, 1990, pp. 85-101.

Punamäki, R. L. "Can Ideological Commitment Protect Children's Psychosocial Well-being in Situations of Political Violence?", Child Development, 67, 1996, pp. 55-69.

Rädda Bamen. Eva, 14, El Salvador. Children of War Newsletter, 2, 1996. En http://www.rb.se/childwar/ cowl.htm

Rädda Bamen. Child Soldiers. Questions and Answers about Child Soldiers from Save the Children, 19981999. En http://www.rb.se/childwardatabase/cfaq.htm

Rodríguez, C. A. "Adquisición de la moral en un contexto de guerra", Boletín de Psicología, San Salvador, Vol. VI, 24, 1987, pp. 123-134.

Santiago, D. The Harvest of Justice. The Church of El Salvador Ten Years After Romero. Mahwah, N. J.: Paulistic Press, 1993.

Schwarz, E. D. y Perry, B. D. "The Post-traumatic Response in Children and Adolescents", Psychiatric Clinics of North America, 17, 311-326.

Simpson, M. A. "What Went Wrong? Diagnostic and Ethical Problems in Dealing with the Effects of Torture and Repression in South Africa". En R. J. KJeber, C. R. Figley y B. P. Gersons (eds.), Beyond Trauma. Cultural and Societal Dynamics. New York: Plenum Press, 1995, pp. 187-212.

Sisti, E. Salud mental de base. San Salvador: UCA Editores, 1995.

Stroebe, W. y Stroebe, M. “The Social Psychology of Social Support”. En E. T. Higgings y A. W. Kruglanski (eds.), Social Psychology. Handbook of Basic 
Principles. New York: Guilford Press, 1996, pp. 597.621.

Summerfield, D. “Addressing Human Response to War Atrocity. Major Challenges in Research and Practices and the Limitations of Western Psychiatric Models". En R. J. Kleber, C. R. Figley y B. P. Gersons (eds.), Beyond Trauma. Cultural and Societal Dynamics. New York: Plenum Press, 1995, pp. 17-29.

UNICEF. The State of the World's Children 1996. En http://www.unicef.org/sowc96/2csoldrs.htm

UNICEF. Annotated principles and best practice on the prevention of recruitment of children into the armed forces and demobilization and social reintegration of child soldiers in Africa. Cape Town: Africa, 1997. En http://www.rb.se/chilwar/tva_97/draft.htm

Woods, D. Children Bearing Military Arrms in Latin America: A summary Report. Eureka Springs, AK: Center on War \& the Child, 1990 (manuscrito).

Yehuda, R. y McFarlane, A. "Conflict Between Knowledge About Posttraumatic Stress Disorder and Its Original Conceptual Bias", American Journal of Psychiatry, 12, 1995, pp. 1705-1713. 